\title{
Nonlinear Dynamics Analysis of Multifactor Low Speed Heavy Load Gear System with Temperature Effect Considered
}

Jungang Wang ( $\nabla$ mewjg@ecjtu.edu.cn )

East China JiaoTong University

Zhengang Shan

East China JiaoTong University

Sheng Chen

East China JiaoTong University

\section{Research Article}

Keywords: temperature effect, nonlinear dynamics, bifurcation diagram, largest Lyapunov exponent graph, time-varying meshing stiffness

Posted Date: December 21st, 2021

DOI: https://doi.org/10.21203/rs.3.rs-1143647/v1

License: (9) This work is licensed under a Creative Commons Attribution 4.0 International License. Read Full License 


\title{
Nonlinear dynamics analysis of multifactor low speed heavy load
}

\section{gear system with temperature effect considered}

\author{
Jungang Wang $\cdot$ Zheng'ang Shan $\cdot$ Sheng Chen
}

J. Wang

Key Laboratory of Conveyance and Equipment, Ministry of Education, East China Jiaotong University, Nanchang 330000, People's Republic of China

e-mail: mewjg@ecjtu.edu.cn

Z. Shan

Key Laboratory of Conveyance and Equipment, Ministry of Education, East China Jiaotong University, Nanchang 330000, People's Republic of China

S. Chen

Key Laboratory of Conveyance and Equipment, Ministry of Education, East China Jiaotong University, Nanchang 330000, People's Republic of China

\begin{abstract}
Low-speed and heavy-duty gears will generate a lot of heat during meshing transmission, which will cause thermal deformation of the gears and affect the transmission performance of the gear system. It is of great significance to explore the influence of temperature effects on the nonlinear dynamics of the gear system. Taking the spur gear system as the research object, considering the nonlinear factors such as time-varying meshing stiffness, tooth backlash and comprehensive error, and introducing the influence of temperature change, the nonlinear dynamic model of the gear system is established, using 4 5th order Runge -Kutta algorithm performs simulation calculation on the model, combined with bifurcation diagram, maximum Lyapunov exponent diagram, phase diagram and Poincare section diagram, etc., to analyze the influence of temperature changes and time-varying stiffness coefficients on the motion characteristics of the gear system. The results show that the influence of temperature change on the gear system is related to the value of the time-varying stiffness coefficient. The larger the value, the more obvious the influence of temperature change; the system will show different dynamic response with the change of the time-varying stiffness coefficient, including four states of single-period motion, multiple-period motion, bifurcation and chaotic motion. The relevant conclusions can provide references for the design of gear systems under special working conditions.
\end{abstract}


Keywords: temperature effect; nonlinear dynamics; bifurcation diagram; largest Lyapunov exponent graph; time-varying meshing stiffness.

\section{Introduction}

Gear system is a transmission mechanism with high efficiency, stable operation and high transmission accuracy, which is widely used in aerospace, high-speed train, power generation and other industries. When the gear system is working, nonlinear excitation will cause the system to enter an unstable state of motion, and even lead to an increase in errors and noise, and premature failure of the gear. Therefore, studying the dynamic characteristics of the gear system and analyzing the influence of various parameters on the stability of the system can not only improve the accuracy of the gear system, but also improve the working state of the gear, thereby extending the life of the gear.

In order to study the non-linear dynamic characteristics of the gear system more deeply and improve the transmission efficiency of the gear system, many scholars have introduced new methods and theories to study the generation mechanism of the nonlinear motion state of the gear transmission system and its influencing factors. Chen et al. ${ }^{[1]}$ established a gear model considering the deformation of a single tooth, and compared it with the traditional mechanical model to provide theoretical guidance for the choice of gear dynamics model. Liu et al. ${ }^{[2]}$ proposed a new model that considers sliding friction, which uses wave excitation to demonstrate its different effects on the gear system. Wang et al. ${ }^{[3]}$ established a random vibration equation based on Newton's law, and analyzed the influence of different parameters on the motion characteristics of the gear system under different working conditions. Liu et al. ${ }^{[4]}$ established a multistage planetary gear dynamics model, used the variable-order Geer numerical integration method to non-dimensionalize the equation of motion, and analyzed various internal nonlinear dynamics of the system through dynamic bifurcation diagrams and time domain diagrams behavior. Literature ${ }^{[5,6]}$ respectively analyzed the influence of 
gear damping ratio and crack failure on the motion characteristics of the system. Geng et $\mathrm{al}^{[7]}$ established a six-degree-of-freedom nonlinear dynamics model considering multiple factors and used the Lundgren-Kutta method to obtain bifurcation diagrams to study the bifurcation and chaos phenomena, which provide a basis for the study of the dynamic characteristics of the gear system under actual working conditions. In addition, many scholars have established models for different gears to analyze their motion characteristics, such as Huang et $\mathrm{al}^{[8]}$ for high contact ratio gears to establish a multifactor nonlinear dynamics model to analyze the motion law of the system with parameter variation. In the literature ${ }^{[9-11]}$, a multi-stage planetary gear nonlinear dynamics model was developed to investigate the dynamics of the gear system from different perspectives such as damping coefficient, tooth side clearance, meshing stiffness and impact stresses, respectively. Xiang et $\mathrm{a}^{[12,13]}$ analyzed the dynamic characteristics of the gear system in terms of excitation frequency, support stiffness and gap bifurcation parameters, respectively, and concluded that the support stiffness has a greater influence on the system motion characteristics and a larger gap can make the system more stable. Liu et al ${ }^{[14]}$ established a nonlinear dynamics model considering thermal deformation, studied the effect of temperature on the thermal time-varying mesh stiffness, and analyzed the effect of temperature variation on the dynamic characteristics of the system by root mean square. Zhou et $\mathrm{a}^{[15]}$ developed a six-degreeof-freedom gear model based on the temperature effect and analyzed the effect of temperature variation and excitation frequency on the dynamic characteristics of the system. Previous studies have analyzed the gear dynamics from different aspects, but those considering temperature effects are few and incomplete.

Based on the principle of thermal deformation, this paper establishes a nonlinear dynamic model of a gear system considering the temperature effect, and analyzes the stability of the nonlinear system by bifurcation diagram, maximum Lyapunov exponent diagram, time domain diagram, phase diagram, spectrum diagram and Poincare section diagram to study the influence of temperature change, time-varying mesh stiffness and other factors on the dynamic characteristics of the system. The study of the influence of temperature change, time-varying mesh stiffness and other factors on the dynamic 
characteristics of the system provides a reference for the design and application of gear systems under special working conditions.

\section{Nonlinear dynamics model of multi-factor gear system considering tempe}

\section{rature effect}

Gear system consists of several gear pairs and drive shafts, bearings and other parts of the mechanical system, under the action of dynamic excitation will produce dynamic response, generate vibration and noise, and the system is subject to dynamic excitation is not only related to the structure of the gear itself, geometric characteristics and error state, etc., but also related to the excitation of other parts in the gear system. Therefore, to analyze the nonlinear dynamics of the gear system, it is necessary to start from the system as a whole and establish an overall vibration analysis model.

\subsection{Time-varying meshing stiffness of gears}

The mesh stiffness $\mathrm{k}(\mathrm{t})$ of a gear is a time-dependent periodic function that changes in stiffness at the same frequency as the internal excitation when the same gear pair is meshed $^{[16]}$, and thus can be expressed as a Fourier series expansion of the form:

$$
\bar{k}(\bar{t})=k_{m}+\sum_{i=1}^{\infty} k_{i} \cos \left(i \overline{\omega_{h}} \bar{t}\right)
$$

where $k_{\mathrm{m}}$ is the average meshing stiffness, $\sum_{i=1}^{\infty} k_{i} \cos \left(i \overline{\omega_{h}} \bar{t}\right)(i=1,2,3 \cdots)$ is the harmonic component, and $\overline{\omega_{h}}$ is the meshing frequency of the gear.

If the first-order harmonic component of the stiffness is taken, the time-varying meshing stiffness is :

$$
\bar{k}(\bar{t})=k_{m}+k_{\alpha} \cos \left(\overline{\omega_{h}} \bar{t}\right)
$$

Where, $k_{\alpha}$ is the fluctuation amplitude of gear mesh stiffness.

\subsection{Gear side clearance}

The gear side clearance function is the nonlinear meshing force displacement 
function of the gear in the presence of side clearance ${ }^{[17]}$, which is a segmented function. Assuming a side gap of $2 \mathrm{~b}$, the segmentation function $\bar{f}(\bar{x}(\bar{t}))$ can be expressed as:

$$
f(\bar{x}(\bar{t}))=\frac{\bar{f}(\bar{x}(\bar{t}))}{k_{m}}=\left\{\begin{array}{cc}
\bar{x}(\bar{t})-b & \bar{x}(\bar{t})>b \\
0 & |\bar{x}(\bar{t})|<b \\
\bar{x}(\bar{t})+b & \bar{x}(\bar{t})<-b
\end{array}\right.
$$

If the gap is symmetric, then $\bar{f}(\bar{x}(\bar{t}))$ is shown in Figure 1 .

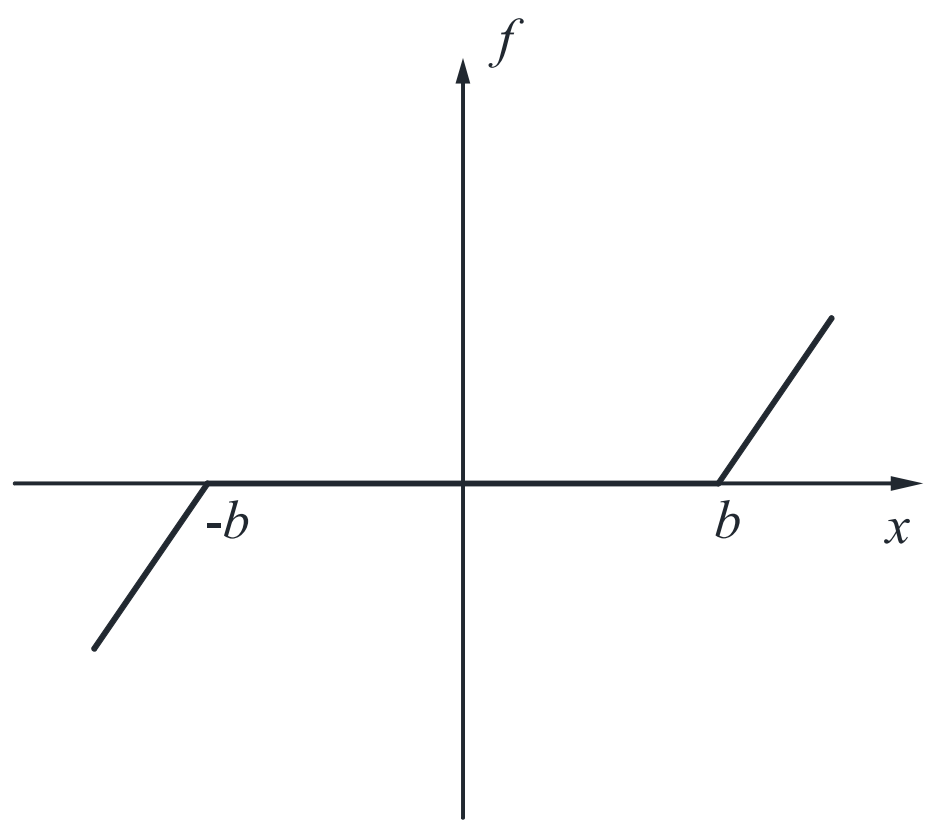

Fig. 1 Nonlinear function of tooth flank clearance

The parameter change of the gear pair will cause the movement process of the gear to change, and the temperature change will cause the gear backlash to change in response. For spur gears, the reduction of the tooth side clearance $\Delta \mathrm{b}_{1}$ caused by the temperature change of the gear is ${ }^{[15]}$ :

$$
\Delta b_{1}=\Delta T_{1} \gamma_{1}\left(1+\Delta T_{1} \gamma_{1}\right) m \pi+\left[m \Delta T_{1} \gamma_{1}\left(z_{1}+z_{2}\right) \sin \alpha\right] / 2
$$

Where: $z_{1}$ and $z_{2}$ are respectively the number of teeth of the master and driven wheels; $m$ is the gear module; $\alpha^{\prime}$ is the meshing angle after thermal deformation, $\alpha^{\prime}=$ $\arccos \left(a \cos \alpha / a^{\prime}\right) ; \Delta T_{1}$ is the temperature rise of the gear; $\gamma_{1}$ is the linear expansion coefficient of the gear.

Therefore, under the influence of temperature changes, the tooth flank clearance is redefined as:

$$
2 b^{\prime}=2 b-\Delta b_{1}
$$

In the formula: $2 b^{\prime}$ is the tooth side clearance considering temperature changes. 
Then the side clearance function $\bar{f}(\bar{x}(\bar{t}))$ including the temperature effect is:

$$
f^{\prime}(\bar{x}(\bar{t}))=\left\{\begin{array}{cc}
\bar{x}(\bar{t})-b^{\prime} & \bar{x}(\bar{t})>b^{\prime} \\
0 & |\bar{x}(\bar{t})|<b^{\prime} \\
\bar{x}(\bar{t})+b^{\prime} & \bar{x}(\bar{t})<-b^{\prime}
\end{array}\right.
$$

Gear meshing error can be expressed as Fourier series:

$$
\bar{e}(\bar{t})=e_{m}+\sum_{h=1}^{\infty}\left[e_{2 h} \cos \left(n \omega_{h} t\right)+e_{2 h+1} \sin \left(n \omega_{h} t\right)\right]
$$

Where $e_{\mathrm{m}}$ is the average meshing error. When only the first order is considered, and $e_{\mathrm{m}}=0$, the meshing error expression can be simplified to:

$$
\bar{e}(\bar{t})=e_{a} \cos \left(\omega_{h}+\varphi_{h}\right)
$$

Where $e_{\mathrm{a}}$ is the error amplitude.

\subsection{Model establishment}

In the working process of the gear system, the overall mass is concentrated, so the concentrated mass method can be used to establish the nonlinear dynamics model of the gear system. The main method is to equate the gear system as a mass with only mass without considering elasticity and a spring with only elasticity without considering mass. It is assumed that the rigidity of the shaft in the gear system is large enough; the friction effect of the bearing is not considered; the direction of the force always acts on the meshing line. The dynamic model of a pair of cylindrical gear pairs is shown in Figure 2: 


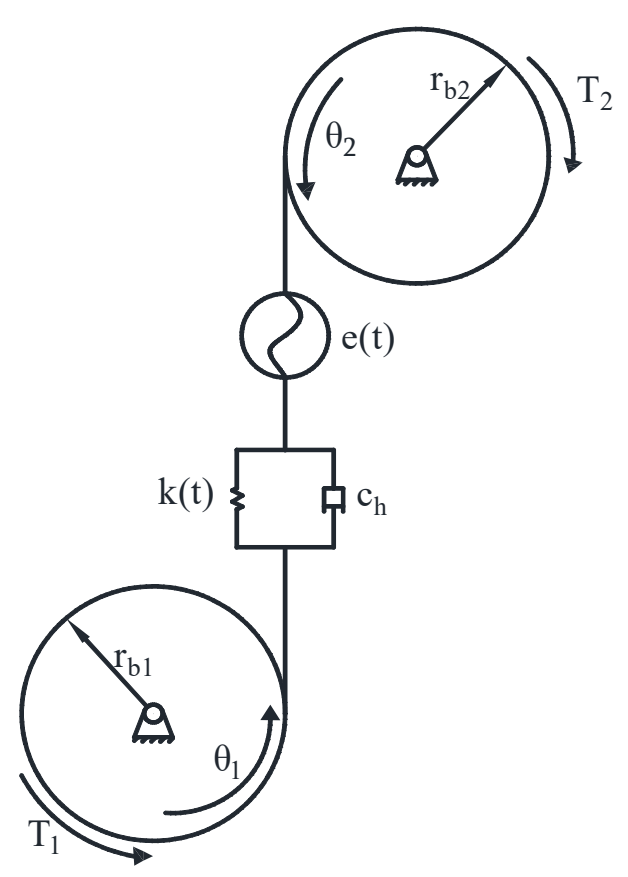

Figure 2 Dynamic model of spur gear system

In the figure: $r_{\mathrm{b} 1}$ and $r_{\mathrm{b} 2}$ are respectively the base circle radius of the master and follower wheels; $T_{1}$ and $T_{2}$ are the torque received by the main and driven wheels respectively; $\theta_{1}$ and $\theta_{2}$ are the torsional vibration displacements of the two gears respectively; $e(\mathrm{t})$ is the static transmission error of gear meshing; $c \mathrm{~h}$ is the damping coefficient in gear meshing; $k(\mathrm{t})$ is the time-varying meshing stiffness of the gear.

According to the dynamic model of the gear system shown in the figure, from Newton's laws of mechanics, the differential equation of motion of the system model can be obtained as:

$$
\left\{\begin{array}{c}
\bar{T}_{1}=I_{1} \ddot{\theta}_{1}+r_{b 1} c_{h}\left[r_{b 1} \dot{\theta}_{1}-r_{b 2} \dot{\theta_{2}}-\dot{\bar{e}}(\bar{t})\right]+\bar{k}(\bar{t}) r_{b 1} \bar{f}(\bar{x}(\bar{t}))\left(r_{b 1} \theta_{1}-r_{b 2} \theta_{2}-\bar{e}(\bar{t})\right) \\
-\bar{T}_{2}=I_{2} \ddot{\theta}_{2}-r_{b 2} c_{h}\left[r_{b 1} \dot{\theta}_{1}-r_{b 2} \dot{\theta}_{2}-\dot{\bar{e}}(\bar{t})\right]-\bar{k}(\bar{t}) r_{b 2} \bar{f}(\bar{x}(\bar{t}))\left(r_{b 1} \theta_{1}-r_{b 2} \theta_{2}-\bar{e}(\bar{t})\right)
\end{array}\right.
$$

In the formula, $\overline{T_{1}}$ and $\overline{T_{2}}$ are the external torque acting on the driving and driven gear; $I_{1}$ and $I_{2}$ are the moments of inertia of the driving and driven gear.

In order to facilitate the calculation and study of the dynamic characteristics of the gear system, the dynamic equations need to be dimensionlessly processed. Define the dimensionless time as $t=\omega_{n} \bar{t}$, and introduce the nominal displacement scale $b$, then other variables can be defined by $\bar{t}$ and $b_{\mathrm{c}}$, assuming that the average meshing stiffness remains unchanged. Then make: 


$$
\overline{\omega_{h}}=\frac{\omega_{h}}{\omega_{0}}, \overline{\omega_{m}}=\frac{\omega_{m}}{\omega_{0}}, \quad F_{m}=\frac{\overline{F_{m}}}{b_{c} m_{e} \omega_{0}^{2}}, \quad F_{h}=\frac{\tilde{e}}{b_{c} m_{e} \omega_{0}^{2}}, \quad \xi=\frac{c_{h}}{2 m_{e} \omega_{0}}, \quad \varepsilon=\frac{k_{a}}{k_{m}}
$$

From this, the dimensionless nonlinear dynamics analysis model of the gear system considering the temperature effect can be derived as:

$$
\ddot{x}(t)+2 \xi \dot{x}(t)+\left[1+\varepsilon \cos \omega_{h} t\right] f^{\prime}(x(t))=F_{m}+F_{h} \omega_{h}^{2} \sin \left(\omega_{h} t+\phi_{h}\right)
$$

In the formula, $F_{\mathrm{m}}$ is the average load, $F_{h} \omega_{h}^{2} \sin \left(\omega_{h} t+\phi_{h}\right)$ is the internal load, $f^{\prime}(x(t))$ is the dimensionless nonlinear displacement function considering the temperature response.

\section{Analysis of nonlinear dynamics}

According to the normalized results of the equation dimension, it can be seen that the dynamic model of the gear is a multi-variable nonlinear system, and it is difficult to analyze the influence of various factors on the stability of the system at the same time.

The gear system analyzed in this paper is an involute spur gear transmission system, and the system parameters are selected as follows. Average load $f_{\mathrm{m}}=0.1$, damping ratio $\xi=0.05$, error amplitude $f_{\mathrm{h}}=0.05$, initial tooth side clearance $b=0.5$, and dimensionless frequency $\omega=1$.

\subsection{The influence of temperature on system characteristics}

The working environment of the gear system is more complicated, and there are many external influence factors. For example, when the gear is operating at high speed, the working temperature of the gear system will increase due to power loss; In addition, the gearboxes are designed according to normal temperature conditions, but the ambient temperature in different regions is different, so it is necessary to analyze the influence of temperature changes on the gear system.

The bifurcation diagram and the maximum Lyapunov exponent diagram are reliable tool diagrams for observing the dynamic response of the system. When the time-varying stiffness coefficient $s$ is small, take $s=0.1$, take the temperature rise of the 
gear as the control parameter, and the bifurcation diagram and the maximum Lyapunov exponent diagram of the gear system when the temperature rise changes from $20^{\circ} \mathrm{C}$ to $200^{\circ} \mathrm{C}$ are shown in Figure 3.

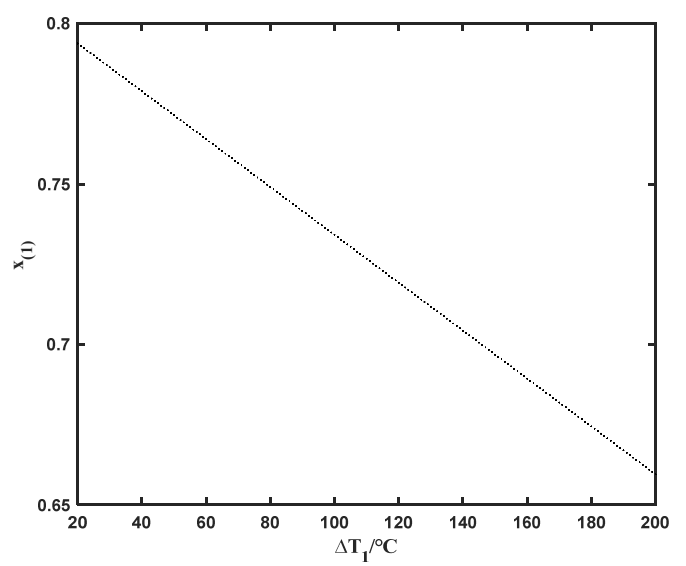

Figure 3 (a) Bifurcation diagram of the gear system with

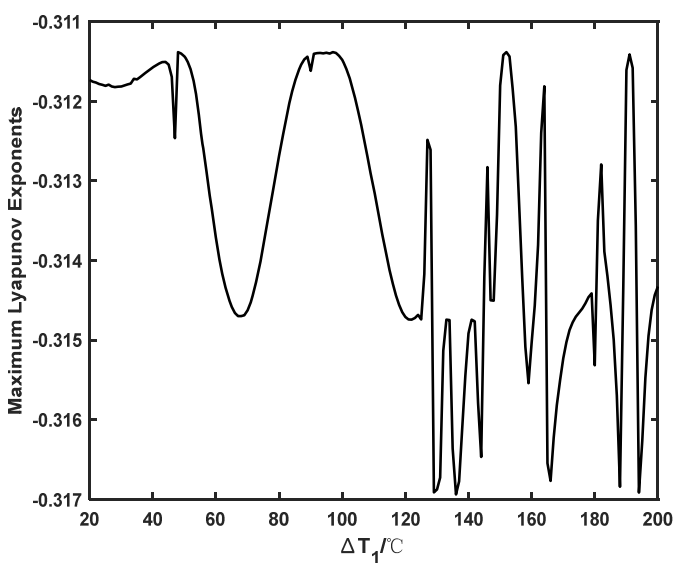

(b) Maximum Lyapunov exponent diagram gear temperature $(\mathrm{s}=0.1)$

As shown in the figure, when the time-varying stiffness coefficient is small, the kinematic characteristics of the gear system are relatively stable with the constant change of temperature, and it has been maintained in a state of cyclic motion, and its maximum Lyapunov exponent has been kept in a negative state overall. The temperature rise is now taken as $50^{\circ} \mathrm{C}$. The simulation obtains the time domain diagram, phase diagram, spectrum diagram and Poincaré cross section of the gear system, as shown in Figure 4. As can be seen from Figure 4, when the temperature rise is $50^{\circ} \mathrm{C}$, the time domain diagram of the gear system is a periodic curve with regular peaks, the phase diagram is a closed single loop, the spectrum diagram shows an isolated peak straight line, and the Poincaré cross-sectional diagram aggregates to a single point, indicating that the system is in a stable single-fold periodic motion. The results show that when the time-varying stiffness coefficient is small, the temperature has less influence on the system characteristics, and the gear system keeps single-fold cycle motion with the change of gear temperature rise. 


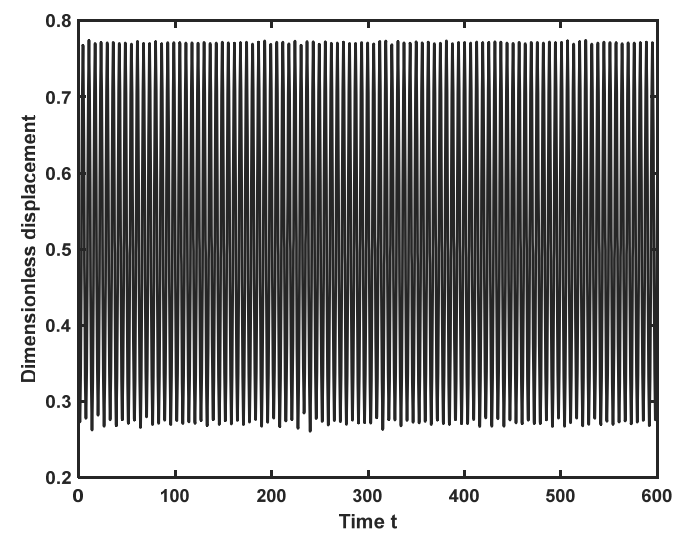

(a) Time domain diagram

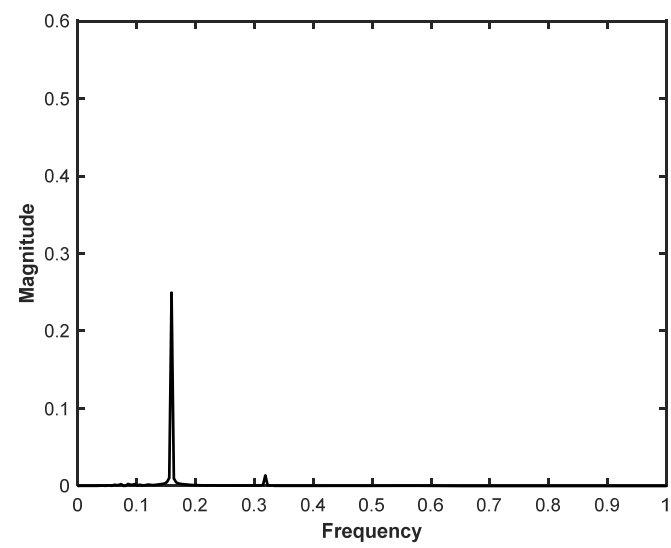

(c) FFT spectrogram

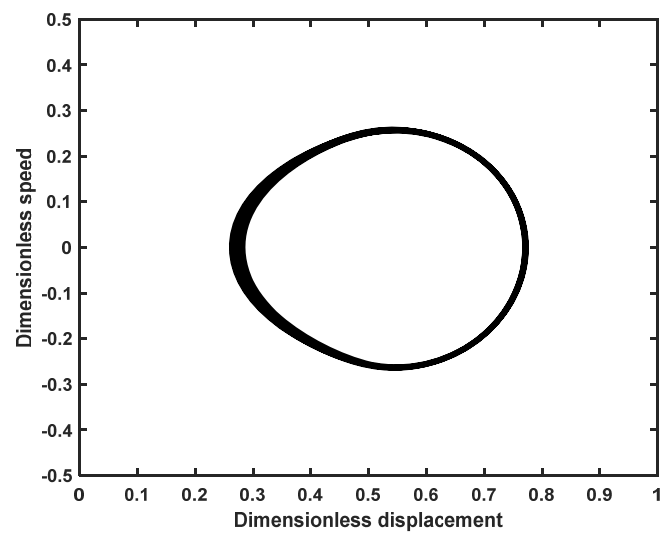

(b) Phase diagram

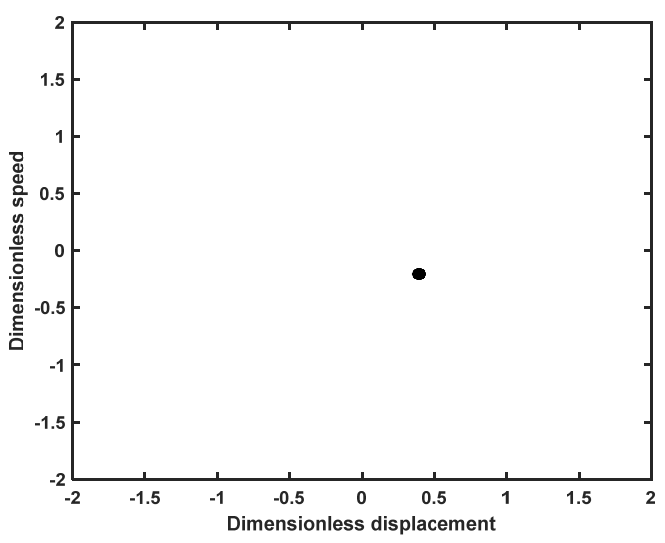

(d) Poincare diagram

Figure 4 Motion characteristics of the gear system at a temperature rise of $50^{\circ} \mathrm{C}(\mathrm{s}=0.1)$

When the time-varying stiffness coefficient $s$ is large, $s=0.6$ is taken, and other parameters of the system are kept constant to obtain the bifurcation diagram and the maximum Lyapunov index diagram of the gear system when the gear temperature rise varies from $20^{\circ} \mathrm{C}$ to $200^{\circ} \mathrm{C}$, as shown in Figure 5 .

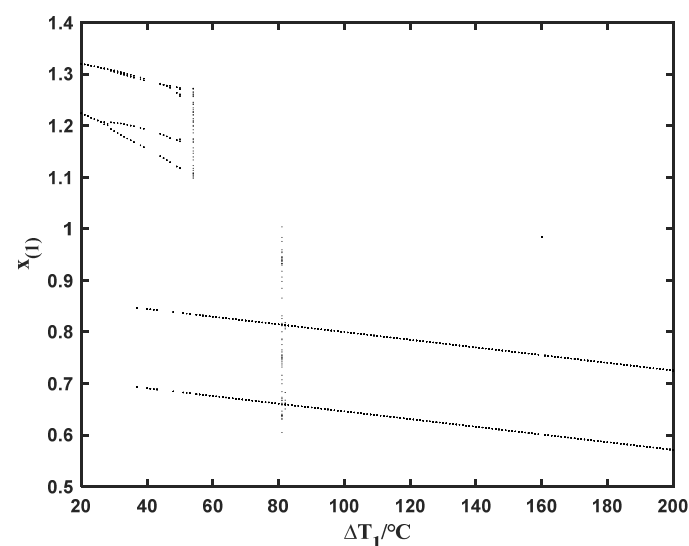

Figure 5 (a) Bifurcation diagram of the gear system

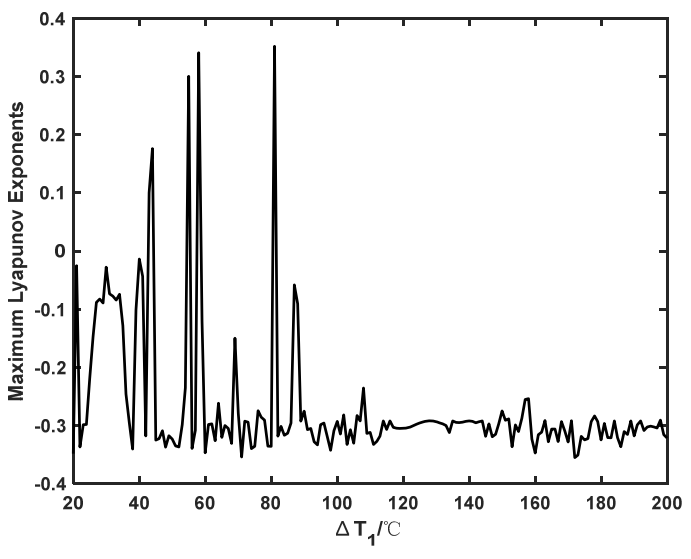

(b) Maximum Lyapunov exponent diagram with gear temperature $(\mathrm{s}=0.6)$ 
As shown in Figure 5, the gear system presents a state of 2 times the proposed periodic motion when the gear temperature rise $\Delta \mathrm{T}_{1}<26^{\circ} \mathrm{C}$ for a time-varying stiffness factor $\mathrm{s}=0.6$. As can be seen from Figure 6 , when the temperature rise $\Delta \mathrm{T}_{1}=20{ }^{\circ} \mathrm{C}$, the time domain diagram is presented as a periodic curve with regular peaks, the phase diagram is a closed curve band with a certain width, and the Poincare diagram is composed of 2 point sets, so the system is in a state of 2 times the proposed periodic motion at this time. In Figure 5, the gear system first bifurcates from 2 times the proposed periodic motion to 4 times the proposed periodic motion as the temperature rise of the gear increases. The time domain diagram, phase diagram and Poincare section diagram at $\Delta \mathrm{T}_{1}=42^{\circ} \mathrm{C}$ are shown in Figure 7. The time domain diagram shows a periodic curve with regular peaks, the phase diagram is a closed curve band, and the Poincare diagram consists of four point sets, so the system is in a 4-fold proposed periodic motion at this time. Then the system enters into the state of 2 times of periodic motion, Figure 8 shows the time domain diagram and phase diagram of the gear system when $\Delta \mathrm{T}_{1}=120{ }^{\circ} \mathrm{C}$, etc. The time domain diagram shows a periodic curve with regular peaks, and the phase diagram is a closed curve band, and there are only two points on the Poincare section diagram, so the system is 2 times of periodic motion at this time. As can be seen, the variation of the system motion characteristics with gear temperature rise obtained in Figures 6-8 remains consistent with the results presented in the bifurcation diagram and the maximum Lyapunov exponent diagram shown in Figure 5.

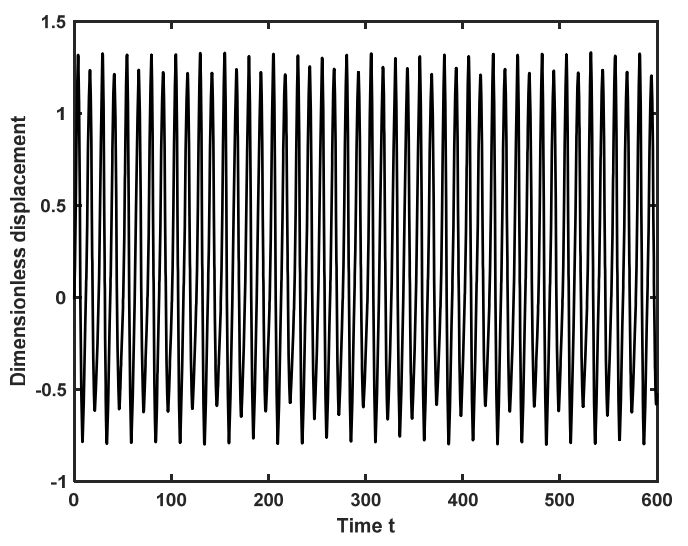

(a) Time domain diagram

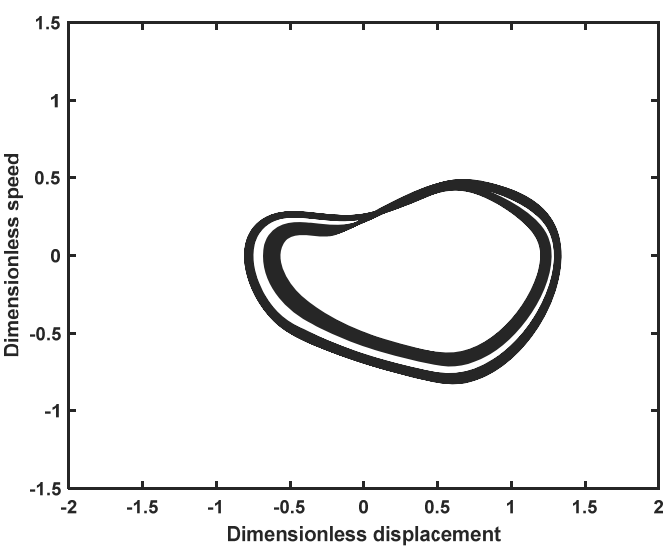

(b) Phase diagram 


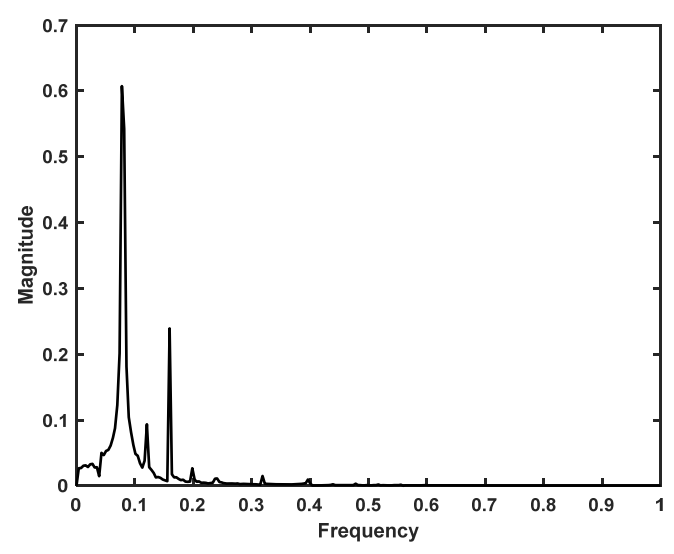

(c) FFT spectrogram

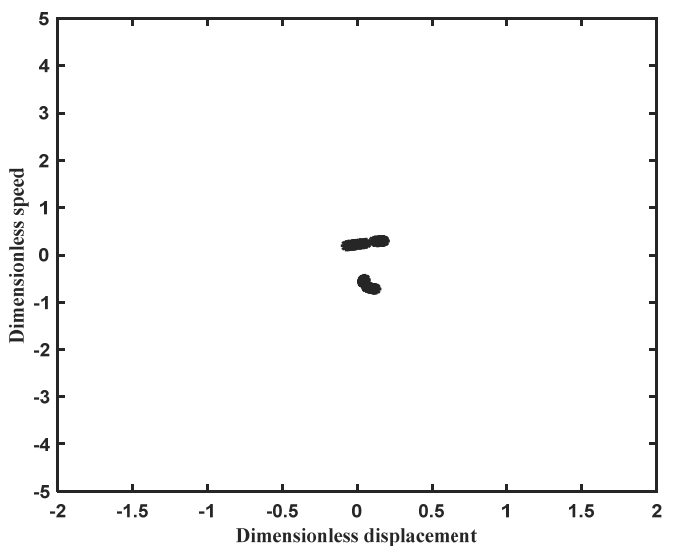

(d) Poincare diagram

Figure 6 Motion characteristics of the gear system at a temperature rise of $20^{\circ} \mathrm{C}(\mathrm{s}=0.6)$

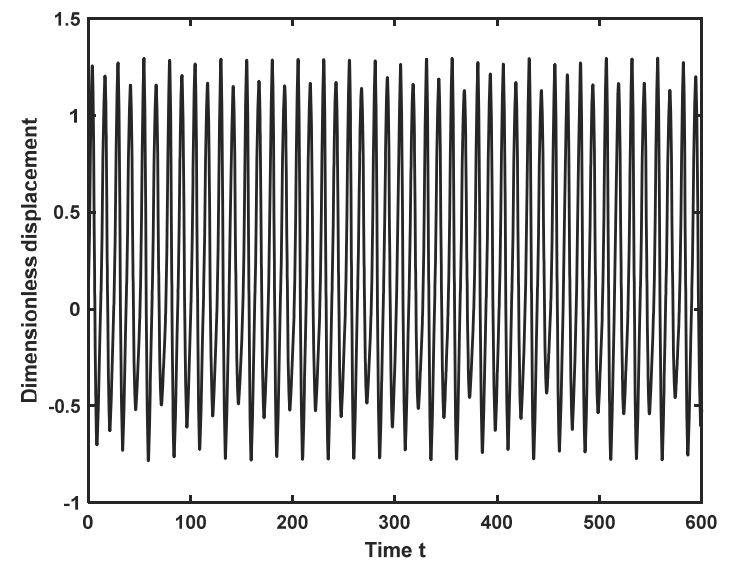

(a) Time domain diagram

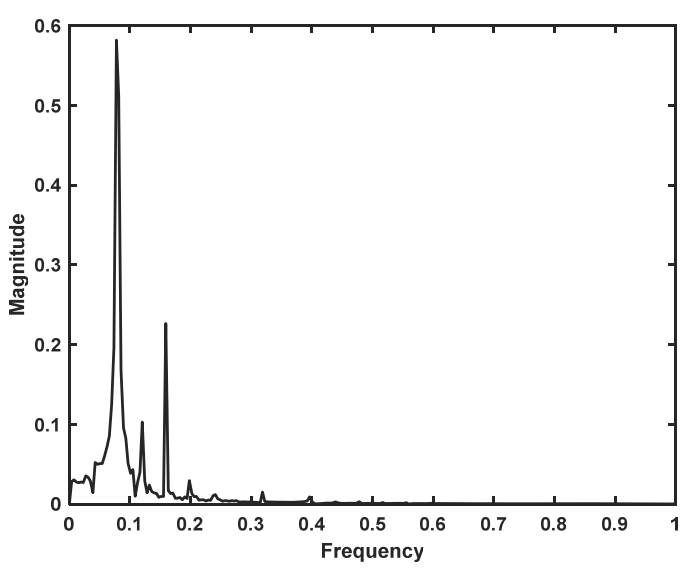

(c) FFT spectrogram

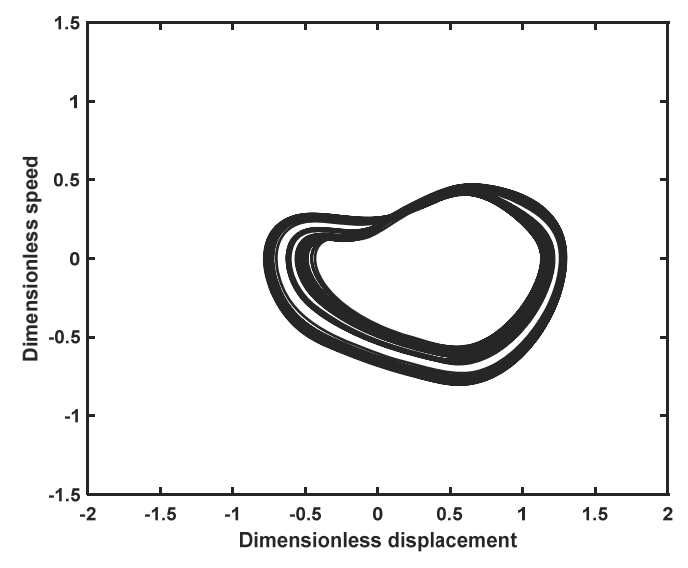

(b) Phase diagram

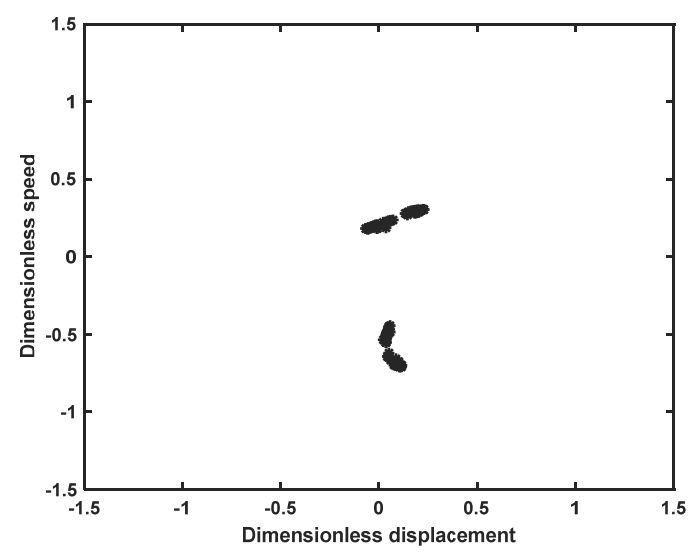

(d) Poincare diagram

Figure 7 Motion characteristics of the gear system at a temperature rise of $42^{\circ} \mathrm{C}(\mathrm{s}=0.6)$ 


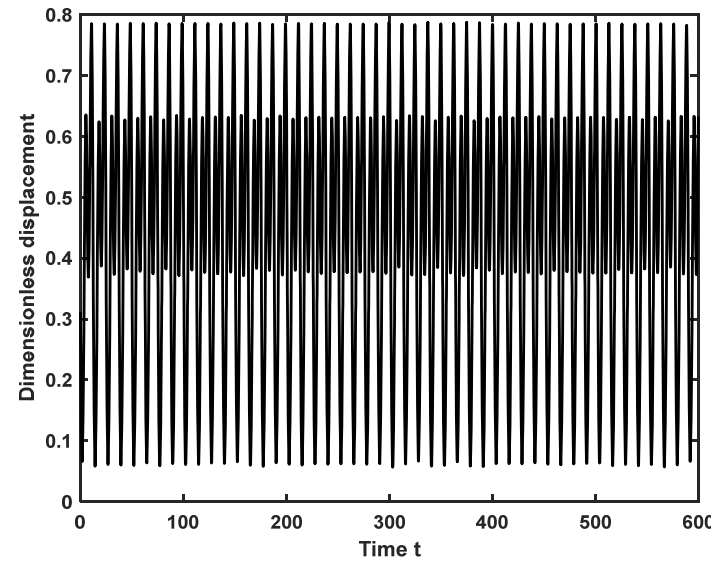

(a) Time domain diagram

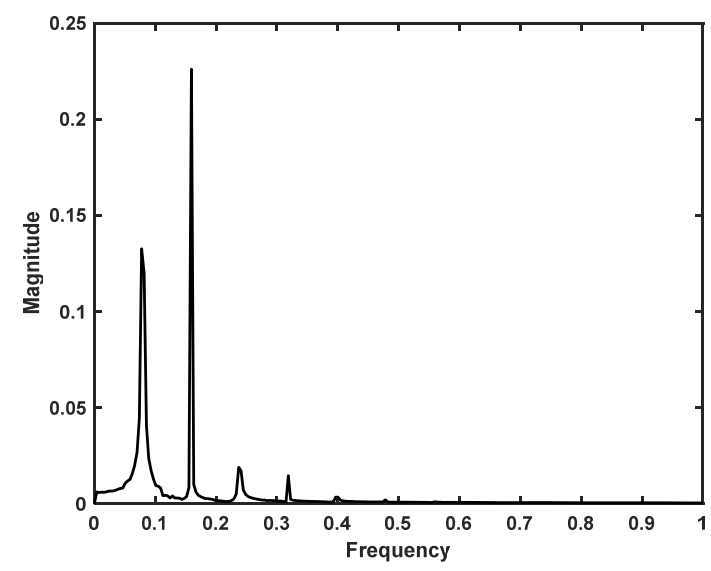

(c) FFT spectrogram

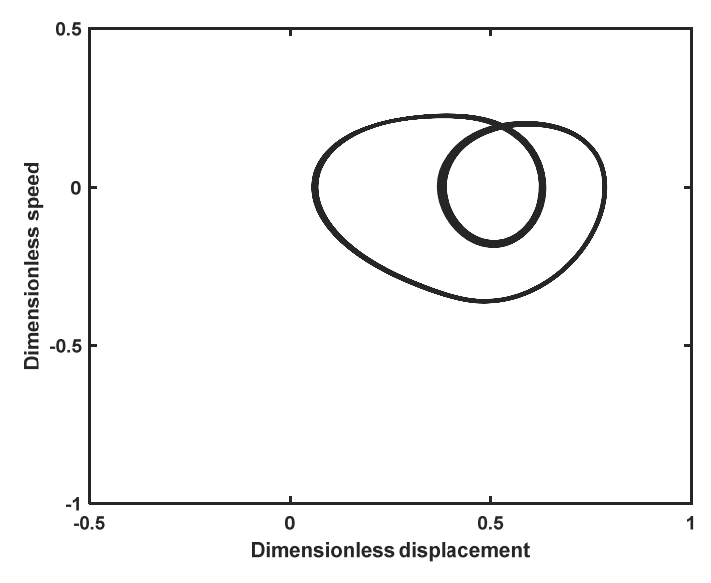

(b) Phase diagram

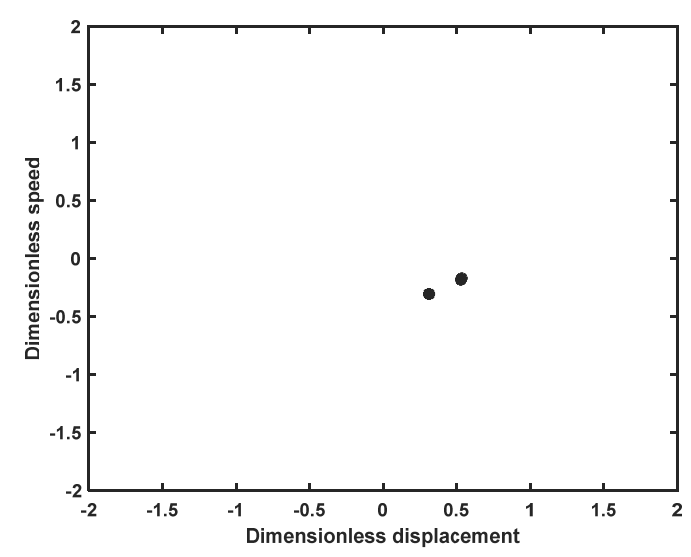

(d) Poincare diagram

Figure 8 Motion characteristics of the gear system at a temperature rise of $120^{\circ} \mathrm{C}(\mathrm{s}=0.6)$

The results show that the temperature variation has a greater impact on the motion characteristics of the gear system when the time-varying stiffness factor is large, and the gear system undergoes a "2 times-4 times-2 times " cyclic motion variation with the temperature rise.

From the above analysis, it can be obtained that the change of gear temperature has different effects on the system motion characteristics under different time-varying stiffness coefficients. Therefore, for some gear systems with special working conditions, reasonable gear system parameters are very important for the system motion characteristics.

\subsection{Effect of time-varying stiffness coefficients on system characteristics}

The time-varying stiffness coefficient $\mathrm{s}$ reflects the variation of the meshing 
stiffness with time and is one of the internal excitation parameters affecting the dynamic characteristics of the gear system. The dynamic characteristics of the gear system are analyzed with the time-varying stiffness coefficient $\mathrm{s}$ as the control parameter, taking the gear temperature rise as $50^{\circ} \mathrm{C}$ and other parameters of the system as constant. Figure 9 shows the bifurcation diagram and the maximum Lyapunov exponent diagram when the time-varying stiffness coefficient $\mathrm{s}$ is varied. Figures $10-14$ show the phase diagram, Poincare mapping and time-domain spectrum of the system when the timevarying stiffness coefficient takes different values, which can explain the dynamics of the gear system in more detail when the time-varying stiffness coefficient is varied.

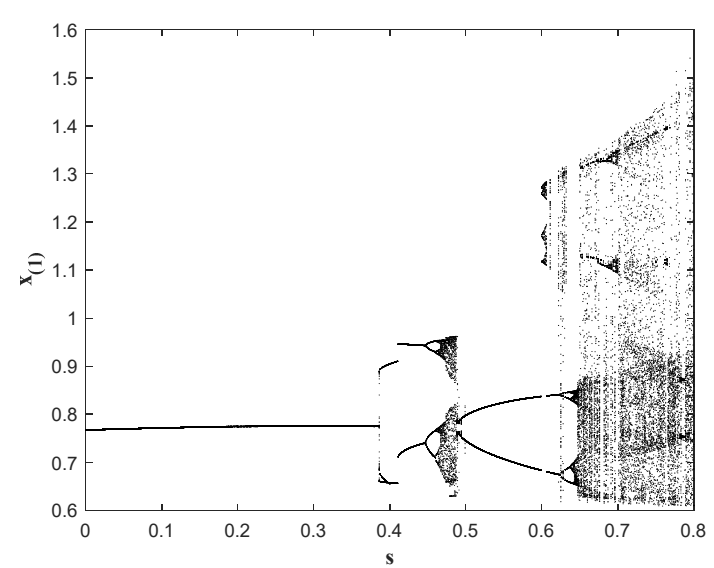

Figure 9(a) Bifurcation diagram of the gear system for the variation of the readily variable stiffness coefficient $s\left(\Delta \mathrm{T}_{1}=50^{\circ} \mathrm{C}\right)$

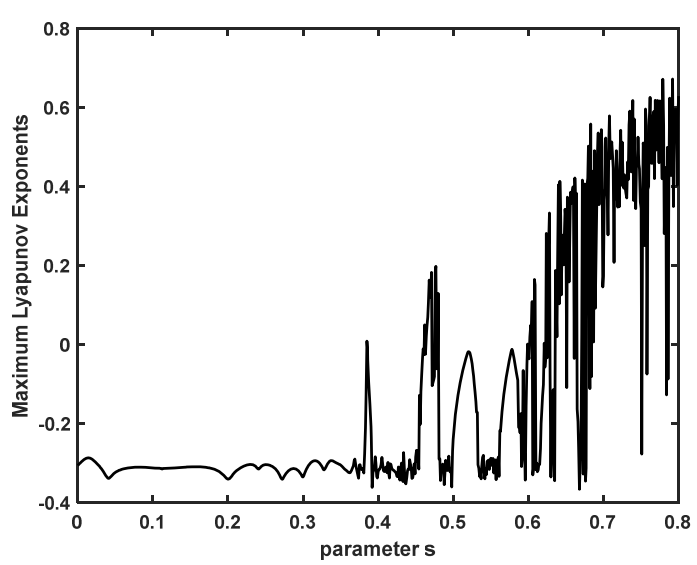

(b) Maximum Lyapunov exponent diagram

As shown in Figure 9, the gear system presents different states of motion when the time-varying stiffness factor $s$ is varied. As the time-varying stiffness coefficient $s$ increases from 0 to 0.386 , the gear system is in single-cycle motion, when its maximum Lyapunov exponent is less than 0 . Figure 10 shows the time domain diagram, phase diagram and Poincare section diagram at $s=0.2$. The time domain diagram presents a periodic curve with regular peaks, the phase diagram is a closed curve, and the Poincare section diagram shows a point, which indicates that the system is in a single-fold periodic motion at this time. When $s>0.386$, the gear system first enters a short period 3 motion, then becomes a period 2 motion, then changes from a period 2 to a period 4 motion, and the maximum Lyapunov exponent also undergoes a "negative-zero- 
negative-positive" change. The kinematic characteristics at $s=0.39$ are shown in Figure 11. The time domain diagram is a periodic curve with regular peaks, the phase diagram has a 3-loop winding closed curve, and the Poincare cross-sectional diagram also has 3 discrete points, indicating that the system is in a periodic 3-motion state at this time. The kinematic characteristics at $s=0.45$ are shown in Figure 12. The phase diagram is a closed curve with a certain width, and the Poincare cross section consists of two point sets, proving that the system is in a 2-fold proposed periodic motion at this time. According to the bifurcation diagram, when the time-varying stiffness coefficient $s>0.464$ increases further, the gear system first enters the chaotic motion state, then enters the 2-fold period motion, and then enters the chaotic state again, and its maximum Lyapunov exponent goes through the change of "positive-zero-negativepositive". When $s=0.56$, the kinematic characteristics of the gear system are shown in Figure 13. The time domain diagram is a periodic curve with regular peaks, the phase diagram has a 2-loop winding closed curve, and the Poincare section diagram has two point sets, so the system is in a 2-fold periodic motion at this time. The time-varying stiffness coefficient $\mathrm{s}$ continues to increase, and when $s=0.75$, the system motion characteristic diagram is shown in Figure 14, and the time-domain diagram is a curve without obvious periodicity, the phase diagram does not repeat and fills a certain closed area, and the Poincare section diagram is composed of patches of dense points, so the system enters a complex chaotic motion phase.

The results show that the time-varying stiffness coefficient has a large influence on the system characteristics, and with the change of the time-varying stiffness coefficient, the gear system has the motion states of single-cycle, multi-cycle and chaotic motion. When the gear system is in a chaotic state, the degree of collision of gears becomes violent, and the long-term chaotic motion will aggravate the gear wear and reduce the gear life, so the appropriate time-varying stiffness coefficient should be selected to avoid entering the chaotic state when working. 


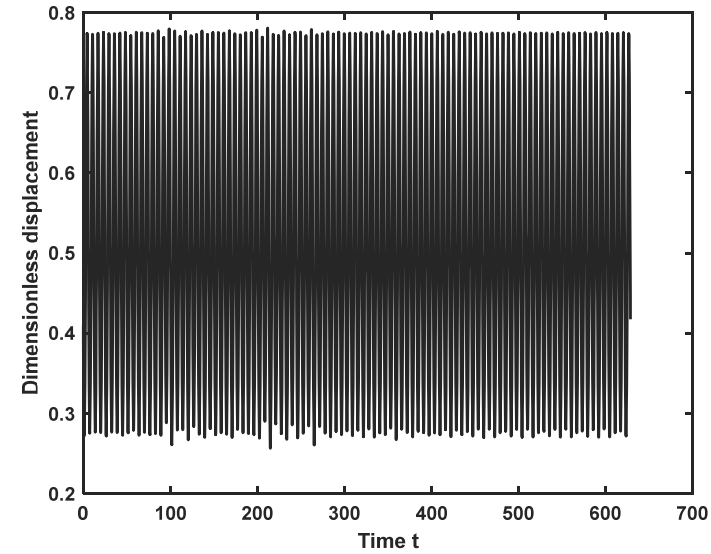

(a) Time domain diagram

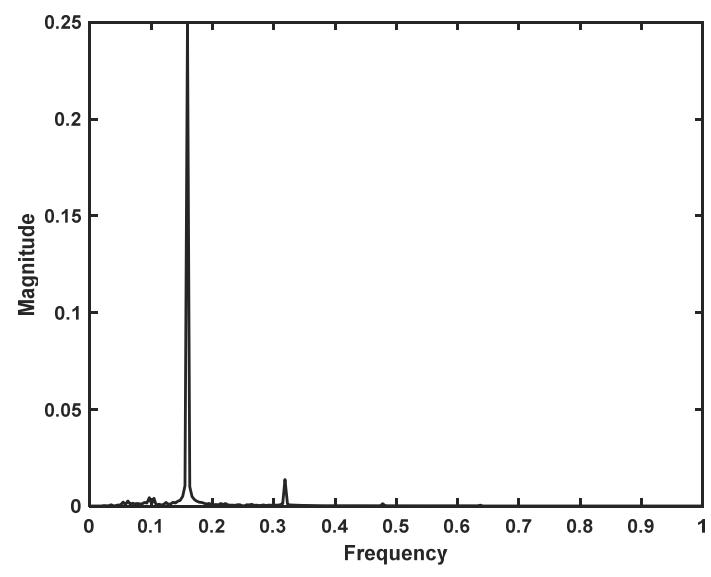

(c) FFT spectrogram

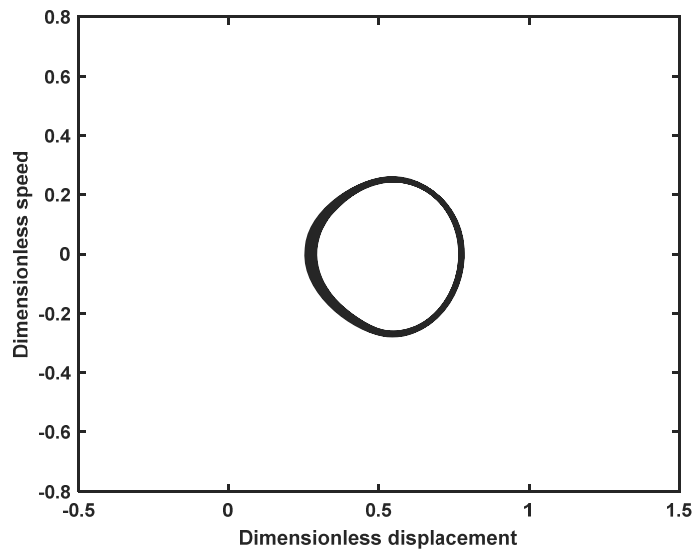

(b) Phase diagram

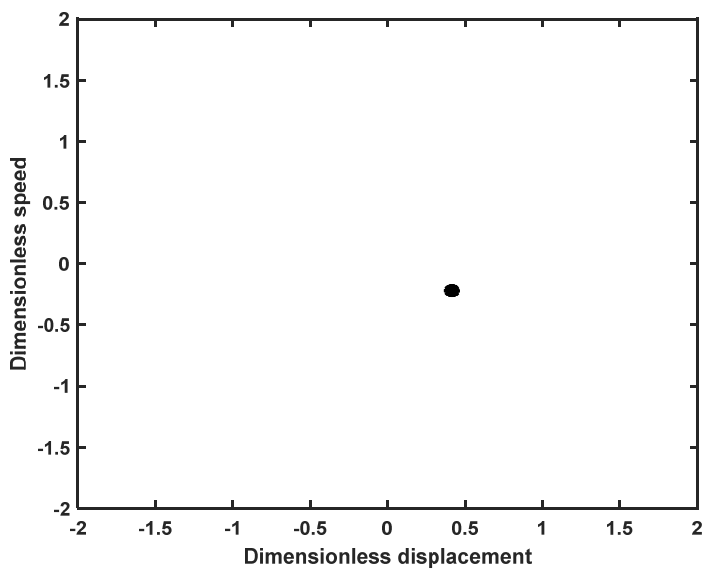

(d) Poincare diagram

Figure 10 Dynamic characteristics of the gear system with time-varying stiffness coefficient $\mathrm{s}=0.2\left(\Delta \mathrm{T}_{1}=50^{\circ} \mathrm{C}\right)$

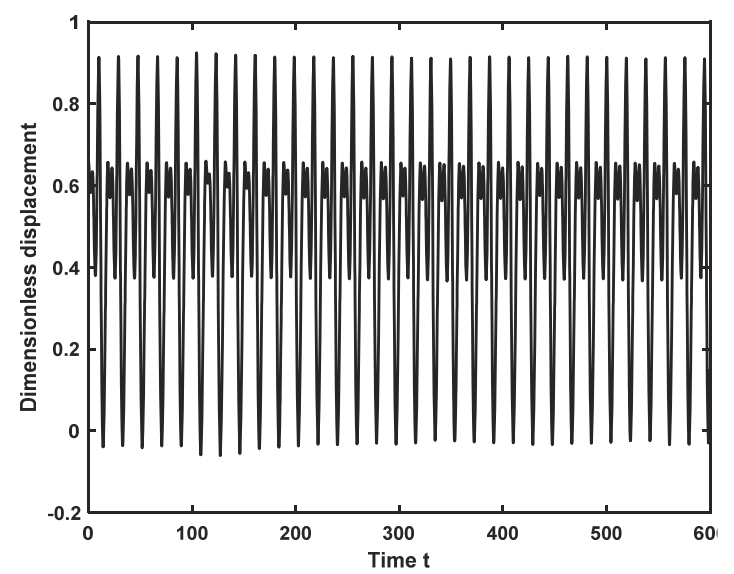

(a) Time domain diagram

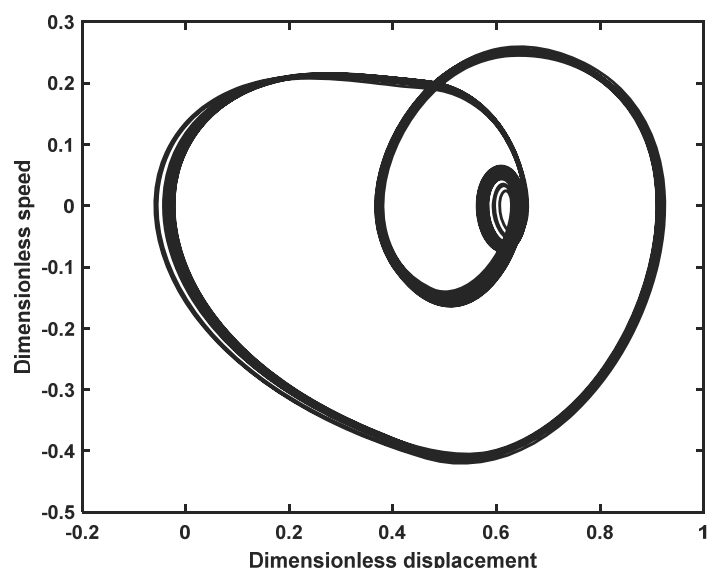

(b) Phase diagram 


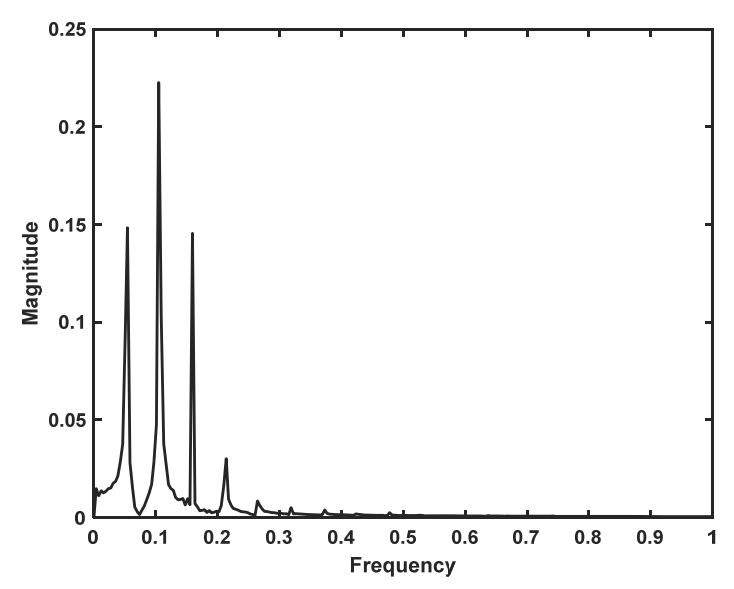

(c) FFT spectrogram

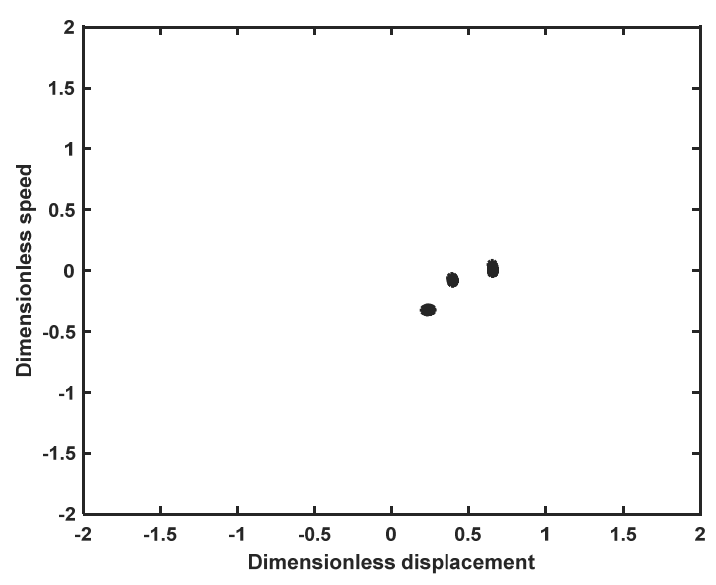

(d) Poincare diagram

Figure 11 Dynamic characteristics of the gear system with time-varying stiffness coefficient $\mathrm{s}=0.39\left(\Delta \mathrm{T}_{1}=50^{\circ} \mathrm{C}\right)$

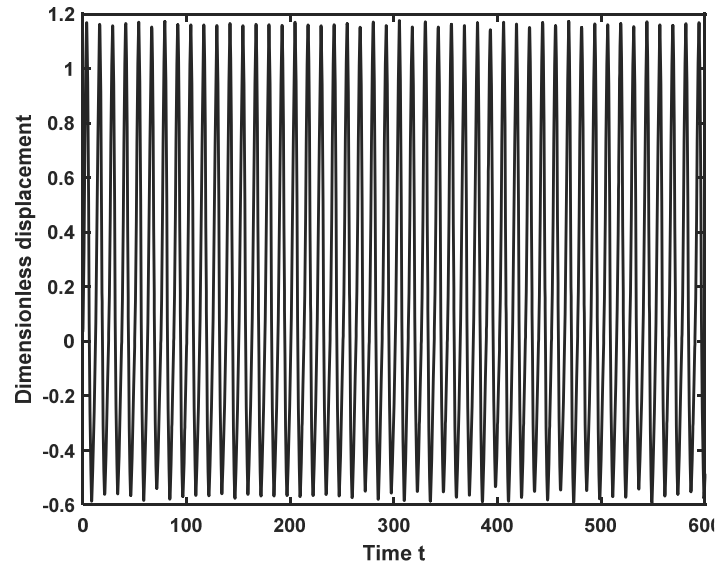

(a) Time domain diagram

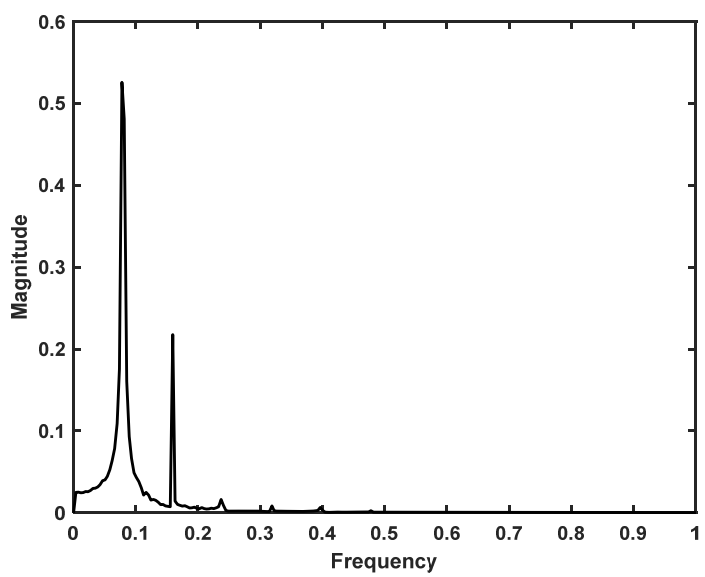

(c) FFT spectrogram

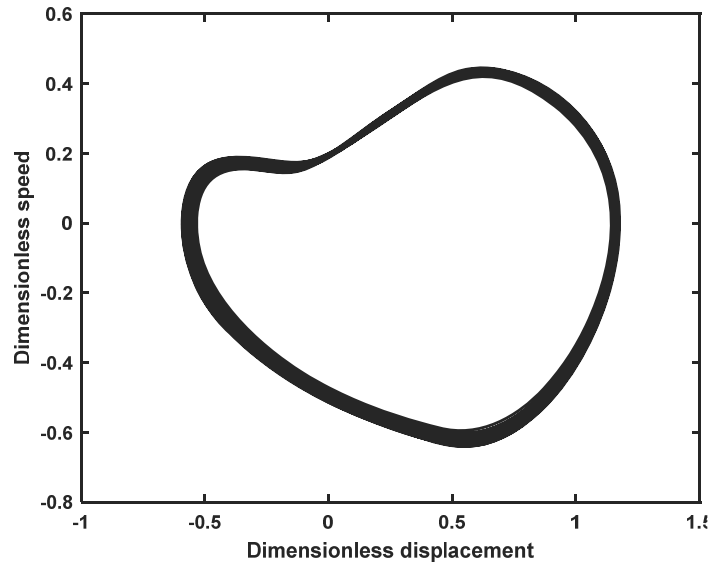

(b) Phase diagram

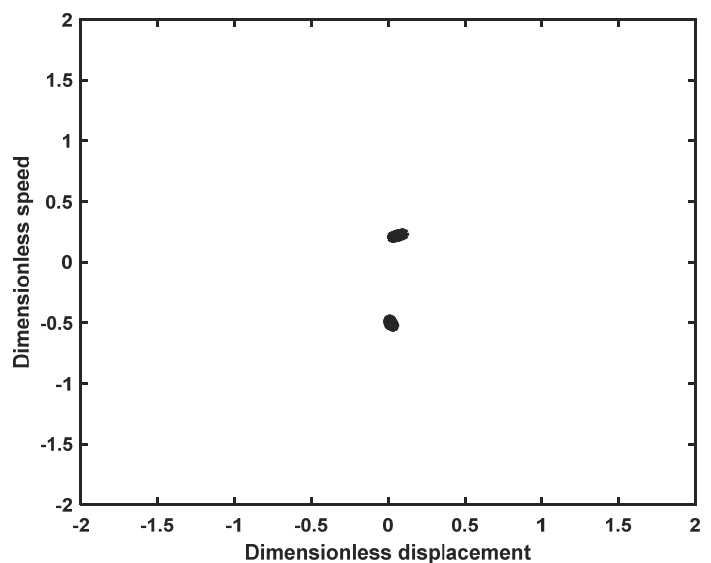

(d) Poincare diagram

Figure 12 Dynamic characteristics of the gear system with time-varying stiffness coefficient $\mathrm{s}=0.45\left(\Delta \mathrm{T}_{1}=50^{\circ} \mathrm{C}\right)$ 


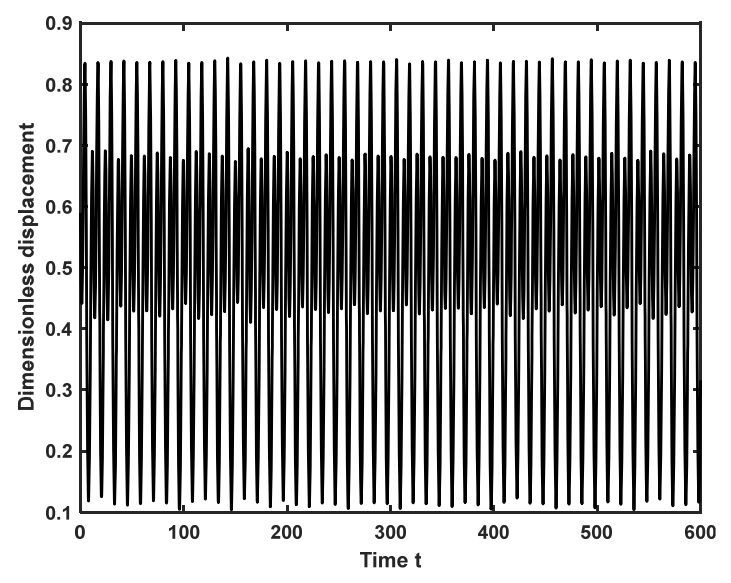

(a) Time domain diagram

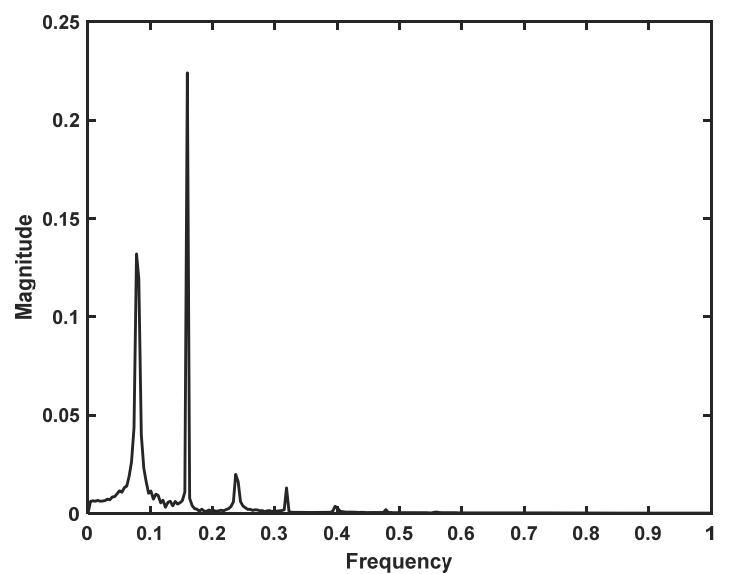

(c) FFT spectrogram

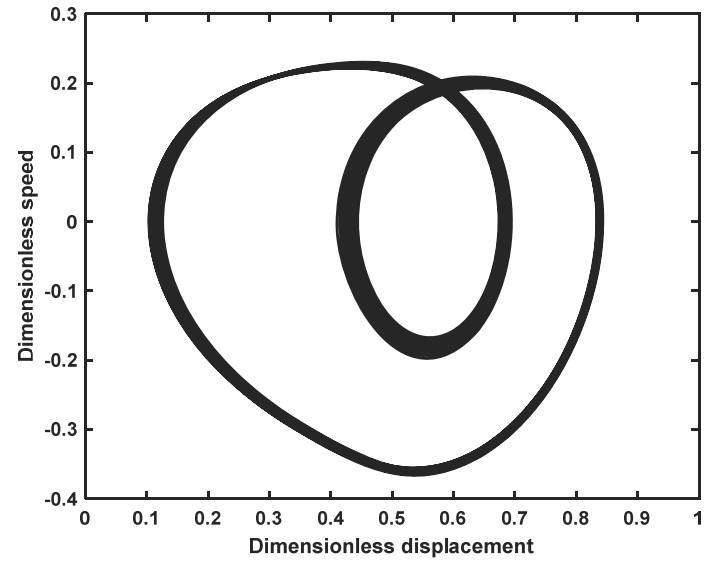

(b) Phase diagram

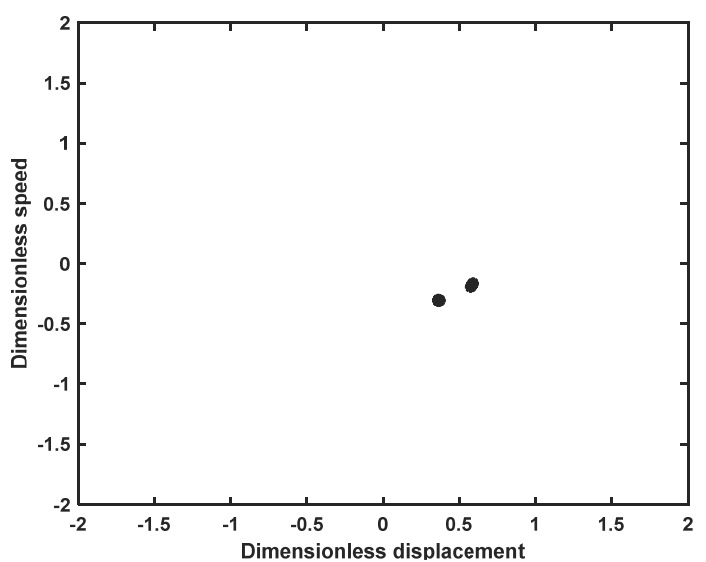

(d) Poincare diagram

Figure 13 Dynamic characteristics of the gear system with time-varying stiffness coefficient $\mathrm{s}=0.56\left(\Delta \mathrm{T}_{1}=50^{\circ} \mathrm{C}\right)$

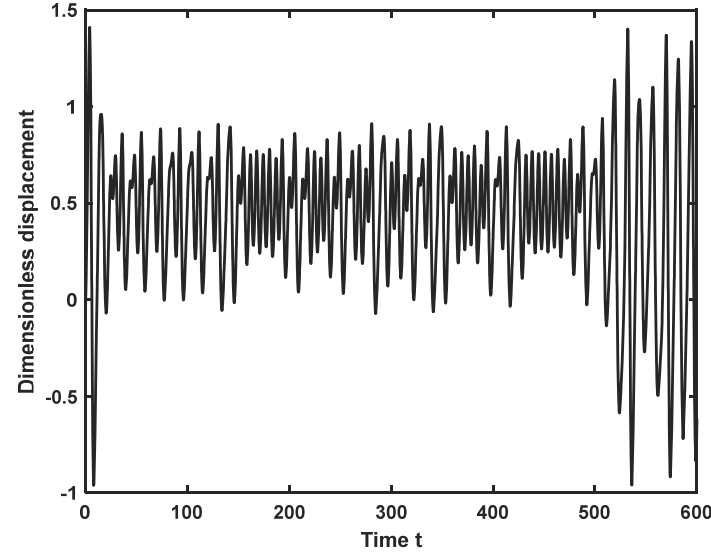

(a) Time domain diagram

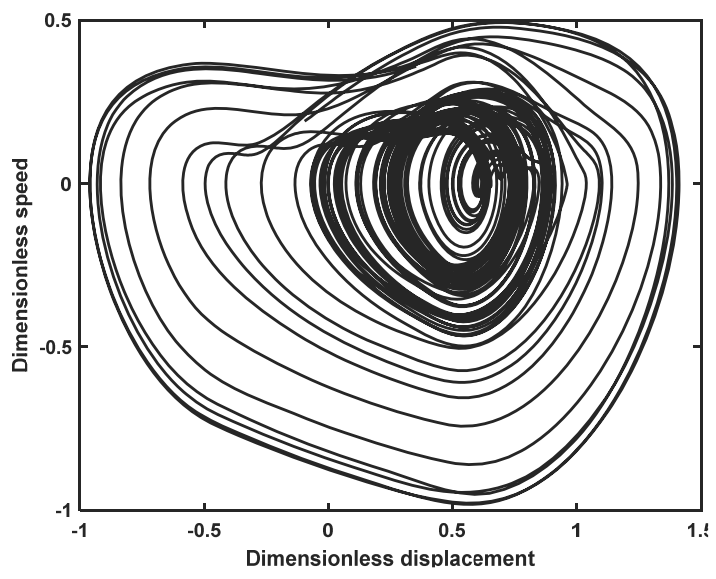

(b) Phase diagram 


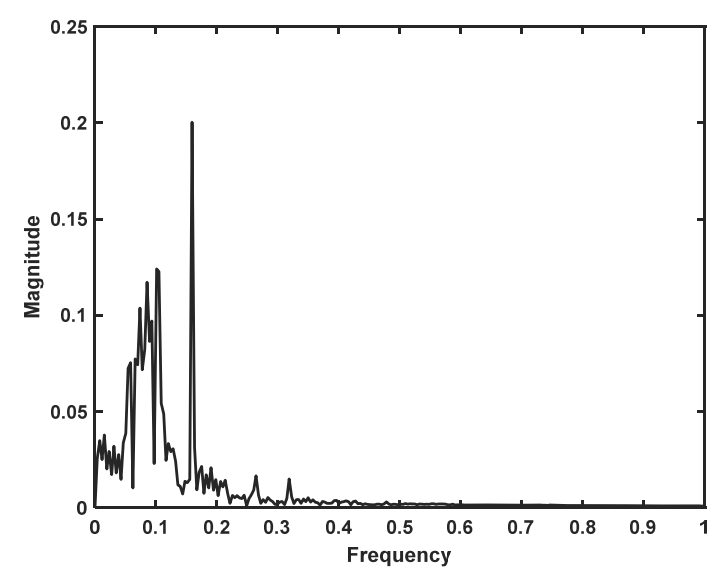

(c) FFT spectrogram

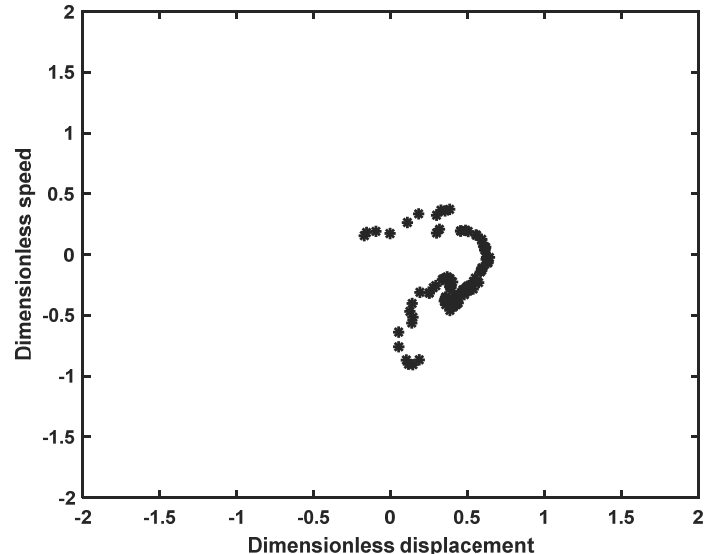

(d) Poincare diagram

Figure 14 Dynamic characteristics of the gear system with time-varying stiffness coefficient $\mathrm{s}=0.75\left(\Delta \mathrm{T}_{1}=50^{\circ} \mathrm{C}\right)$

\section{Conclusion}

In this paper, based on the heat deformation principle, a nonlinear dynamics model of the gear system considering the temperature effect is established, and the stability of the nonlinear system is analyzed by bifurcation diagram, maximum Lyapunov exponent diagram, time domain diagram, phase diagram, spectrum diagram and Poincare cross section diagram, and the following conclusions are obtained.

(1) The simulation reveals the effect law of temperature on the system motion characteristics under different time-varying stiffness coefficients. For the dynamics model established in this paper, when the time-varying stiffness coefficient takes a small value $(s=0.1)$, the effect of temperature change on the system motion is small, and the gear system keeps a stable single-fold cycle motion as the temperature increases. When the time-varying stiffness factor is large $(s=0.6)$, the effect of temperature change on the system motion is larger, and as the temperature increases, the system undergoes a "2 times-4 times-2 times" cycle motion change, and there is a bifurcation phenomenon.

(2) The effect of the time-varying stiffness coefficient on the kinematic characteristics of the gear system at a certain temperature rise $\left(\Delta \mathrm{T}_{1}=50^{\circ} \mathrm{C}\right)$ is analyzed. As the time-varying stiffness coefficient increases, the gear system moves from singleperiod motion to a brief period 3 motion, then to a period 2 motion, then to a period 4 
motion through bifurcation, then to a brief chaotic state, then to a period 2 motion again, and finally to a chaotic state as the time-varying stiffness coefficient continues to increase.

Gear temperature variation affects gear systems with different parameter conditions differently. The simulation results allow the selection of suitable parameter conditions to ensure that the system is in a stable cyclic motion state and to extend the service life of the gear system. This study can provide a reference for the design and application of gear systems under special working conditions.

Funding The authors are grateful for the financial support provided by the National Natural Science Foundation of China (Grant No.51765020), and the Natural Science Foundation of Jiangxi Province (Grant No. 20161BAB206153).

\section{Compliance with ethical standards}

Disclosure statement The authors report there are no competing interests to declare.

Data availability statement The data that support the findings of this study are available from the corresponding author, $\mathrm{Jg} \mathrm{W}$, upon reasonable request. 


\section{References}

[1] Zaigang Chen, Jieyu Ning, Kaiyun Wang, Wanming Zhai. An improved dynamic model of spur gear transmission considering coupling effect between gear neighboring teeth. Nonlinear Dynamics 106(1), 339-357 (2021)

[2] F. H. Liu, H. J. Jiang, S. N. Liu, X. H. Yu. Dynamic behavior analysis of spur gears with constant \& variable excitations considering sliding friction influence. Journal of Mechanical Science and Technology 30(12), 5363-5370 (2016)

[3] J. Y. Wang, H. T. Wang, H. Wang, L. X. Guo. Influence of the Random System Parameters on the Nonlinear Dynamic Characteristics of Gear Transmission System. International Journal of Nonlinear Sciences and Numerical Simulation 18(7-8), 619-630 (2017)

[4] S. Li, Q. M. Wu, Z. Q. Zhang. Bifurcation and chaos analysis of multistage planetary gear train. Nonlinear Dynamics 75(1-2), 217-233 (2014)

[5] Junguo Wang, Zhaoyuan Yao, M. F. Hassan, Yongxiang Zhao. Modeling and dynamics simulation of spur gear system incorporating the effect of lubrication condition and input shaft crack. Engineering Computations ahead-of-print(aheadof-print), (2021)

[6] J. Y. Wang, H. T. Wang, L. X. Guo. Analysis of Stochastic Nonlinear Dynamics in the Gear Transmission System with Backlash. International Journal of Nonlinear Sciences and Numerical Simulation 16(2), 111-121 (2015)

[7] Z. B. Geng, K. Xiao, J. Y. Li, J. X. Wang. Bifurcation and Chaos of a Spur Gear Transmission System With Non-Uniform Wear. Journal of Vibration and Acoustics-Transactions of the Asme 143(3), 11 (2021)

[8] K. Huang, Y. Yi, Y. S. Xiong, Z. B. Cheng, H. Chen. Nonlinear dynamics analysis of high contact ratio gears system with multiple clearances. Journal of the Brazilian Society of Mechanical Sciences and Engineering 42(2), 16 (2020)

[9] L. Xiang, N. Gao, A. J. Hu. Dynamic analysis of a planetary gear system with multiple nonlinear parameters. Journal of Computational and Applied Mathematics 327, 325-340 (2018)

[10] Y. Zhao, M. Ahmat, K. Bari. Nonlinear dynamics modeling and analysis of transmission error of wind turbine planetary gear system. Proceedings of the Institution of Mechanical Engineers Part K-Journal of Multi-Body Dynamics 228(4), 438-448 (2014)

[11] Z. B. Zhu, L. C. Cheng, R. Xu, R. P. Zhu. Impacts of Backlash on Nonlinear Dynamic Characteristic of Encased Differential Planetary Gear Train. Shock and Vibration 2019, 15 (2019)

[12] L. Xiang, Z. Q. Deng, A. J. Hu. Dynamical Analysis of Planetary Gear Transmission System Under Support Stiffness Effects. International Journal of Bifurcation and Chaos 30(6), 13 (2020)

[13] L. Xiang, Y. Zhang, N. Gao, A. J. Hu, J. T. Xing. Nonlinear Dynamics of a Multistage Gear Transmission System with Multi-Clearance. International Journal of Bifurcation and Chaos 28(3), 12 (2018) 
[14] H. Liu, P. F. Yan, P. Gao. Effects of Temperature on the Time-Varying Mesh Stiffness, Vibration Response, and Support Force of a Multi-Stage Planetary Gear. Journal of Vibration and Acoustics-Transactions of the Asme 142(5), 15 (2020)

[15] Zhou Yang, Che Bowen, Xie Zhijie, Li Dalei. Nonlinear dynamic analysis of a multi-degree-of-freedom gear system based on temperature effect. Modern Manufacturing Engineering (05), 1-8 (2020)

[16] Zhang Ju, Liang Shuang, Li Xueyun. Study on nonlinear dynamics of gearbox transmission system considering multiple influencing factors. CHINESE JOURNAL OF APPLIED MECHANICS 38(02), 794-801 (2021)

[17] Wujiu Pan, Liangyu Ling, Haoyong Qu, Minghai Wang. Early Wear Fault Dynamics Analysis Method of Gear Coupled Rotor System Based on Dynamic Fractal Backlash. Journal of Computational and Nonlinear Dynamics 17(1), (2021) 